Submitted to the Annals of Statistics arXiv: 1312.1200

\title{
SUPPLEMENTARY MATERIAL FOR: ESTIMATING A PROBABILITY MASS FUNCTION WITH UNKNOWN LABELS
}

\author{
By Dragi Anevski *, Richard D. Gill ${ }^{\dagger}$ And Stefan Zohren $\ddagger$ \\ Lund University* ${ }^{*}$ Leiden University ${ }^{\dagger}$ and University of Oxford ${ }^{\ddagger}$
}

\begin{abstract}
This is the supplementary material for, Anevski, Gill and Zohren, "Estimating a probability mass function with unknown labels" submitted to the Annals of Statistics, which in this document is referred to as the main article.
\end{abstract}

\section{APPENDIX A: EXISTENCE OF THE EXTENDED MODEL NONPARAMETRIC MAXIMUM LIKELIHOOD ESTIMATOR}

We first give a simple demonstration of non-existence of the MLE in the basic model. Thus define

$$
\widehat{\theta}=\arg \max _{\theta: \theta_{1} \geq \theta_{2} \geq \ldots, \sum_{\alpha=1}^{\infty} \theta_{\alpha}=1} \sum_{\chi} \prod_{i} \theta_{\chi(i)}^{N_{i}}
$$

Assume $n=2$ and the partition $N=(1,1)$ The data give a likelihood

$$
\sum_{\chi} \prod_{i=1}^{2} \theta_{\chi(i)}^{N_{i}}=\theta_{1} \theta_{2}+\theta_{1} \theta_{3}+\ldots+\theta_{2} \theta_{3}+\theta_{2} \theta_{4}+\ldots
$$

with $\chi$ a bijection as specified in the main article, noting the tie-breaking rule than $N_{i}=N_{j}$ implies $\chi(i)<\chi(j)$. We see first that there can only be a solution if all $\theta$ 's are equal. In fact, writing the likelihood as

$$
\theta_{1} \theta_{2}+\theta_{1}\left(1-\left(\theta_{1}+\theta_{2}\right)\right)+\theta_{2}\left(1-\left(\theta_{1}+\theta_{2}\right)\right)+R
$$

where $R$ contains all terms with only indices 3 and higher, and differentiating w.r.t. $\theta_{1}$ we see that there is a maximum if and only if $\theta_{2}+1-2 \theta_{1}-\theta_{2}-\theta_{2}=0$, i.e. if and only if $1-2 \theta_{1}-\theta_{2}=0$. Since the likelihood is symmetric in the parameters, we get that there is a maximum if and only if $1-2 \theta_{i}-\theta_{j}=0$ for all $i \neq j$, which is only possible if all $\theta_{i}=\theta_{j}$. But if the cardinality $|\mathcal{A}|$ of

MSC 2010 subject classifications: 62G05, 62G20, 65C60, 62P10 
the species names is infinite, the restrictions $\theta_{i}=\theta_{j}, i \neq j, \sum_{\alpha \in \mathcal{A}} \theta_{\alpha}=1$, are not satisfied for any choice of parameters. Therefore there is no (ordered) $\theta$ that maximizes the likelihood in this case. If $|\mathcal{A}|=: \aleph<\infty$ however then there is a solution which clearly is $\hat{\theta}=(1 / \aleph, \ldots, 1 / \aleph) \in[0,1]^{\aleph}$.

Next we prove that the PML in the extended model always exists, as stated in Theorem 1 in the main article. Recall the definition of the (PML) as

$$
\widehat{\theta}=\arg \max _{\theta: \theta_{1} \geq \theta_{2} \geq \ldots, \sum_{\alpha=1}^{\infty} \theta_{\alpha} \leq 1} \sum_{\chi} \frac{n !}{N_{0} ! \prod_{i \geq 1} N_{i} !} \theta_{0}^{N_{0}} \prod_{\alpha=1}^{\infty} \theta_{\alpha}^{N_{\chi^{-1}(\alpha)}},
$$

with $N_{0}=n-\sum_{\alpha=1}^{\infty} N_{\chi^{-1}(\alpha)}$ and with the mappings $\chi: \mathbb{N} \rightarrow\{0,1, \ldots, \infty\}$ satisfying that for every $\alpha \geq 1$ there exists exactly one $i$ such that $\chi(i)=\alpha$, with tie-breaking rule $N_{i}=N_{j}$ implies $\chi(i)<\chi(j)$, and that $\chi(i)=0$ implies $N_{i}=0$ or 1 . Recall also the definition of the measure $\mathrm{P}^{(n, \phi)}$ for the possibly defective probability $\phi$ : For any set $A$ of partitions of $n$,

$$
\mathrm{P}^{(n, \phi)}(A)=\sum_{\left(N_{1}, N_{2}, \ldots\right) \in A} \sum_{\chi} \frac{n !}{N_{0} ! \prod_{i \geq 1} N_{i} !} \theta_{0}^{N_{0}} \prod_{\alpha=1}^{\infty} \theta_{\alpha}^{N_{\chi-1}(\alpha)},
$$

with $N_{0}=n-\sum_{\alpha=1}^{\infty} N_{\chi^{-1}(\alpha)}$.

Recall that $\Theta$ is given the topology of pointwise convergence. We would like to note that Orlitsky et al. [2] suggested that the $\ell_{2}$-norm does the job in deriving existence, and they are (almost) right.

Proof. (Theorem 1)

(i) To see that $\Theta$ is compact, consider a sequence $\theta^{(m)}$. For given $\alpha$ the sequence of numbers $\theta_{\alpha}^{(m)}$ is bounded, hence contains a convergent subsequence. By a standard diagonalisation argument, we can extract from $\theta^{(m)}$ a subsequence for which each coordinate converges.

(ii) Suppose we take an iid sample of size $n$ of animals of different species labeled $1,2,3, \ldots$. The species have probabilities $\theta_{1} \geq \theta_{2} \geq \ldots$ where $\sum_{k=1}^{\infty} \theta_{k}=1-\theta_{0}$. The index $k=1,2, \ldots$ labels species in (decreasing) order of their probabilities; $k=0$ stands for a "blob" of very many different species each of very small probability. Two different animals each given the species label $k=0$ will always belong to different species.

Let the r.v. $S_{i}$ denote the species label of the $i$ th animal in our sample, $i=1, \ldots, n ; S_{i} \in\{1,2, \ldots\} \cup\{0\}$, and note that $S_{1}, \ldots, S_{n}$ are i.i.d. r.v.'s. Note also that since the (theoretical) species labels are not observed, $S_{1}, \ldots, S_{n}$ are not statistics, they are however random variables. When we have obtained our sample we can determine for any two elements of the sample whether they belong to the same species or not. This determines a 
random equivalence relation on the numbers $\{1,2, \ldots, n\}$, which we shall denote by $\sim: i \sim j$ if and only if $i=j$ or $i \neq j$ and $S_{i}=S_{j} \neq 0$.

We will introduce a second random equivalence relation denoted by $\sim_{K}$ : $i \sim_{K} j$ if and only if $i=j$ or $i \neq j$ and $S_{i}=S_{j} \in\{1, \ldots, K\}$. These equivalence relations determine partitions $\mathcal{P}_{n}$ and $\mathcal{P}_{n}^{K}$ of the set $\{1,2, \ldots, n\}$ into equivalence classes, so e.g. $\mathcal{P}=\left\{H_{1}, \ldots, H_{\tilde{n}}\right\}$, with

$$
\{1, \ldots, n\}=\cup_{j=1}^{\tilde{n}} H_{j},
$$

with $H_{i} \cap H_{j}=\emptyset$ if $i \neq j$. Note that the equivalence relation $\sim_{K}$ is stricter than the equivalence relation $\sim$, in the sense that $i \sim_{K} j \Rightarrow i \sim j$, which implies that the partition $\mathcal{P}_{n}{ }^{K}$ generated by $\sim_{K}$ is finer than the partition $\mathcal{P}_{n}$ generated by $\sim$, i.e. an equivalence set in $\mathcal{P}_{n}$ is a union of equivalence sets of $\mathcal{P}_{n}^{K}$.

The sizes of the equivalence classes determine partitions $n=\left|H_{1}\right|+\ldots+$ $\left|H_{\tilde{n}}\right|$, in the number theoretic sense, of the number $n$. Let $\Pi_{n}$ denote the random partition of the number $n$ generated by $\sim$ and $\Pi_{n}^{K}$ that generated by $\sim_{K}$. Denote by $\pi_{n}$ and $\pi_{n}^{K}$ possible realisations of both. Denote by $P_{\theta}$ the probability measure induced by $\theta=\left(\theta_{1}, \theta_{2}, \ldots\right)$. Now given a parameter vector $\theta$ define $\theta^{K}=\left(\theta_{1}, \ldots, \theta_{K}, 0,0, \ldots\right)$. All species with label larger than $K$ have been merged with the blob.

Define the event

$$
A_{n, K}=\cup_{1 \leq i<j \leq n}\left(\left\{S_{i}=S_{j}\right\} \cap\left\{S_{i}>K\right\} \cap\left\{S_{j}>K\right\}\right),
$$

of at least two animals in the sample belong to the same species and have a species label larger than $K$. The complement is

$$
A_{n, K}^{c}=\cap_{1 \leq i<j \leq n}\left(\left\{S_{i} \neq S_{j}\right\} \cup\left\{S_{i} \leq K\right\} \cup\left\{S_{j} \leq K\right\}\right)
$$

i.e. the event that for every pair of animals no two are from the same species or at least one of the pair of animals has a label smaller than or equal to $K$. Note that

$$
\begin{aligned}
P_{\theta}\left(A_{n, K}\right) & \leq \frac{1}{2} n(n-1) \sum_{i=K+1}^{\infty} \theta_{j}^{2} \\
& \leq \frac{1}{2} n(n-1) \theta_{K+1} \sum_{i=K+1}^{\infty} \theta_{j} \\
& \leq \frac{1}{2} n(n-1) \theta_{K+1},
\end{aligned}
$$

where the first inequality follows by Boole's inequality and since the $S_{i}$ are i.i.d., and the second since $\theta_{k} \leq \theta_{K+1}$ for $k \geq K+1$. 
We have that $\Pi_{n}=\Pi_{n}^{K}$ on $A_{n, K}^{c}$. In fact, on $A_{n, K}^{c}$, let $1 \leq i<j \leq n$ be fixed but arbitrary. Then, if $S_{i} \neq S_{j}$ both of $i \sim j$ and $i \sim_{K} j$ are violated so then $i, j$ are not in the same partition in $\mathcal{P}_{n}$ nor in $\mathcal{P}_{n}^{K}$. If instead $S_{i}=S_{j}$ then we must have that $S_{j} \leq K$ and $S_{j} \leq K$, and then if $S_{i}=S_{j}>0$ both $i \sim j$ and $i \sim_{K} j$ are satisfied so then $i, j$ are in the same partition in both $\mathcal{P}_{n}$ and $\mathcal{P}_{n}^{K}$, and if $S_{i}=S_{j}=0$ neither of $i \sim j, i \sim_{K} j$ are satisfied and then $i, j$ are not in the same partition in $\mathcal{P}_{n}$ nor in $\mathcal{P}_{n}^{K}$. Since this holds for every $i<j$, and since $\Pi_{n}$ and $\Pi_{n}^{K}$ are counting the sizes of the partitions in $\mathcal{P}_{n}$ and $\mathcal{P}_{n}^{K}$, we have shown that on $A_{n, K}^{c}$ the two partitions $\Pi_{n}$ and $\Pi_{n}^{K}$ of $n$, coincide. It therefore follows that for any given partition $\pi_{n}$ of the number $n$

$$
\begin{aligned}
P_{\theta}\left(\Pi_{n}=\pi_{n}\right) & =P_{\theta}\left(\left\{\Pi_{n}=\pi_{n}\right\} \cap A_{n, K}^{\mathrm{c}}\right)+P_{\theta}\left(\left\{\Pi_{n}=\pi_{n}\right\} \cap A_{n, K}\right) \\
& \leq P_{\theta}\left(\left\{\Pi_{n}^{K}=\pi_{n}\right\} \cap A_{n, K}^{\mathrm{c}}\right)+P_{\theta}\left(A_{n, K}\right) \\
& \leq P_{\theta}\left(\left\{\Pi_{n}^{K}=\pi_{n}\right\}\right)+P_{\theta}\left(A_{n, K}\right) \\
& \leq P_{\theta^{K}}\left(\left\{\Pi_{n}=\pi_{n}\right\}\right)+\frac{1}{2} n(n-1) \theta_{K+1} .
\end{aligned}
$$

Let $\theta^{(m)}$ be a sequence of parameter vectors converging coordinatewise to $\theta$ as $m \rightarrow \infty$, and let $\pi_{n}$ be a fixed partition of the number $n$. We want to prove that $P_{\theta^{(m)}}\left(\Pi_{n}=\pi_{n}\right) \rightarrow P_{\theta}\left(\Pi_{n}=\pi_{n}\right)$ as $m \rightarrow \infty$.

Let $\delta>0$. Then there is finite $K=K(\delta)$ such that

$$
\frac{1}{2} n(n-1) \theta_{K+1} \leq \frac{1}{2} \delta .
$$

Furthermore there is a finite $M=M\left(\delta, \theta_{K+1}\right)$ such that if $m>M$, we have that

$$
\frac{1}{2} n(n-1) \theta_{K+1}^{(m)} \leq \frac{\delta}{2}
$$

Therefore, using the inequality(4) with $\theta$ replaced by $\theta^{(m)}$,

$$
P_{\theta^{(m)}}\left(\Pi_{n}=\pi_{n}\right) \leq P_{\theta^{(m) K}}\left(\left\{\Pi_{n}=\pi_{n}\right\}\right)+\frac{\delta}{2} .
$$

Now $P_{\theta^{(m) K}}\left(\left\{\Pi_{n}=\pi_{n}\right\}\right) \rightarrow P_{\theta^{K}}\left(\left\{\Pi_{n}=\pi_{n}\right\}\right)$ as $m \rightarrow \infty$, since $\theta^{(m) K}$ only contains finitely many non-zero coordinates. This implies that

$$
\limsup _{m \rightarrow \infty} P_{\theta^{(m)}}\left(\Pi_{n}=\pi_{n}\right) \leq P_{\theta^{K}}\left(\left\{\Pi_{n}=\pi_{n}\right\}\right)+\frac{\delta}{2} .
$$


Next, we get

$$
\begin{aligned}
P_{\theta}\left(\left\{\Pi_{n}=\pi_{n}\right\}\right) & \geq P_{\theta}\left(\left\{\Pi_{n}=\pi_{n}\right\} \cap A_{n, K}^{c}\right) \\
& =P_{\theta}\left(\left\{\Pi_{n}^{K}=\pi_{n}\right\} \cap A_{n, K}^{c}\right) \\
& =P_{\theta}\left(\left\{\Pi_{n}^{K}=\pi_{n}\right\}\right)-P_{\theta}\left(\left\{\Pi_{n}^{K}=\pi_{n}\right\} \cap A_{n, K}\right) \\
& \geq P_{\theta^{K}}\left(\left\{\Pi_{n}=\pi_{n}\right\}\right)-\frac{1}{2} n(n-1) \theta_{K},
\end{aligned}
$$

where the first equality follows since $\Pi_{n}^{K}=\Pi_{n}$ on $A_{n, K}^{c}$, and last inequality follows from (3). Finally (6), (7) and (5) imply that

$$
\limsup _{m \rightarrow \infty} P_{\theta^{(m)}}\left(\Pi_{n}=\pi_{n}\right) \leq P_{\theta}\left(\left\{\Pi_{n}=\pi_{n}\right\}\right)+\delta .
$$

To show a lower bound for the liminf, use of (7) with $\theta^{K}$ replaced by $\theta^{(m) K}$, and noting that $\theta_{K+1}^{K}=0$, gives

$$
P_{\theta^{(m) K}}\left(\Pi_{n}=\pi_{n}\right) \leq P_{\theta^{(m)}}\left(\Pi_{n}=\pi_{n}\right) .
$$

Thus

$$
\begin{aligned}
P_{\theta^{(m)}}\left(\Pi_{n}=\pi_{n}\right) & \geq P_{\theta^{(m) K}}\left(\Pi_{n}=\pi_{n}\right) \\
& \rightarrow P_{\theta^{K}}\left(\Pi_{n}=\pi_{n}\right) \\
& \geq P_{\theta}\left(\Pi_{n}=\pi_{n}\right)-\frac{1}{2} \delta
\end{aligned}
$$

where the first inequality holds by (8), then the limit (which is a liminf) is taken as $m \rightarrow \infty$ and the last inequality follows by (4) and (5).

Thus lim sup and lim inf of $P_{\theta^{(m)}}\left(\Pi_{n}=\pi_{n}\right)$ are within $\delta$ of $P_{\theta}\left(\Pi_{n}=\pi_{n}\right)$. Since $\delta>0$ was arbitrary it follows that $P_{\theta}\left(\Pi_{n}=\pi_{n}\right)$ is the limit as $m \rightarrow \infty$ of $P_{\theta^{(m)}}\left(\Pi_{n}=\pi_{n}\right)$.

\section{APPENDIX B: COMPUTATION OF THE NONPARAMETRIC MAXIMUM LIKELIHOOD ESTIMATOR}

In this appendix we discuss an implementation of data, of the likelihood and the Stochastic Approximation EM algorithm (SAEM) used to calculate the NPMLE introduced in the previous section, in particular, the sieved model defined in Equation (7) of the main article. 
B.1. The sample. After reduction by sufficiency, the data can be represented by the partition of the sample-size $T$, in the number theoretic sense: A partition of $T$ is a non-increasing sequence of positive integers adding to up $T$, e.g., $T=7=3+2+1+1$. The number of different integers appearing in the partition can be much smaller than the length of the partition itself, and often a more compact representation of $T$ consists of two equal length sequences of positive integers $n_{1}<\cdots<n_{J}$ and $r_{1}, \ldots, r_{J}$ where $n_{j}$ are the distinct numbers occuring in the partition, ordered, $r_{j}$ are the number of repetitions of $n_{j}$ and $J$ is the number of distinct numbers occurring in the partition. Write $\mathbf{r}=\left(r_{j}\right)_{1 \leq j \leq J}$ and $\mathbf{n}=\left(n_{j}\right)_{1 \leq j \leq J}$. In the above example $J=3, \mathbf{n}=(1,2,3)$, and $\mathbf{r}=(2,1,1)$.

Assumption 1. Assume that $n_{1}=1$, i.e. there exist singletons in the sample, and $J \geq 2$, i.e. the sample contains non-singletons.

Assumption 1 is typically satisfied in practice; in the sequel we assume this to hold.

B.2. The population. We will use indices $a, b$, etc. to denote (nonblob) population species, identified by position when ordered by decreasing probability. Different blob species are merged into one group and assigned the index 0 . We suppose the population consists of a finite number $K$ of species of positive probability $p_{1} \geq p_{2} \geq \cdots \geq p_{K}>0$ and a blob of uncountably many species each of zero probability, but together of positive probability $p_{0}=1-\sum_{a=1}^{K} p_{a}>0$. The population species $a, b$ etc. are therefore integers between 0 and $K$ where 0 indicates a blob species and 1 to $K$ a non-blob species.

In some situations one can be interested in the case $K=0$ but this special case is easy to study separately, so we will assume in the sequel $K \geq 1$.

B.3. The likelihoods. The "missing data" consists of the identification of each non-blob population species either with an index $1 \leq j \leq J$ to indicate that this species was indeed observed in the sample, and was one of the $r_{j}$ species observed exactly $n_{j}$ times, or with some kind of marker, we will use the index 0 for this purpose, to indicate that this species was not observed at all.

Under Assumption 1 the number of singletons $r_{1}$ in the sample is positive, and $J \geq 2$, so the sample contains both singletons and non-singletons. Then, the missing data can be represented by a function $\boldsymbol{\psi}:\{1, \ldots, K\} \rightarrow$ $\{0,1, \ldots, J\}$, which satisfies the two constraints

C1: $\sum_{a=1}^{K} 1\{\boldsymbol{\psi}(a)=j\}=r_{j}$, for each $j>1$, 
C2: $\sum_{a=1}^{K} 1\{\boldsymbol{\psi}(a)=1\} \leq r_{1}$.

It is easily seen that $(\mathbf{n}, \mathbf{r}, \boldsymbol{\psi})$ is a sufficient statistic for $\boldsymbol{\theta}$ based on the full data, just as $(\mathbf{n}, \mathbf{r})$ is a sufficient statistic for $\boldsymbol{\theta}$ when we are only given the actually observed data.

Because of the constraints $\mathbf{C 1}, \mathbf{C 2}$, we must have $\sum_{j=2}^{J} r_{j} \leq K$, i.e. the number of non-singleton species observed is not larger than $K$.

Recall that $T=\sum_{j=1}^{J} r_{j} n_{j}$ is the total size of the sample. For given $\boldsymbol{\psi}$, define

$$
n_{0}=r_{1}-\sum_{a=1}^{K} 1\{\boldsymbol{\psi}(a)=1\},
$$

the total number of times a blob species was observed. The full data likelihood is

$$
\frac{T !}{n_{0} ! \prod_{1 \leq a \leq K: \boldsymbol{\psi}(a) \geq 1} n_{\boldsymbol{\psi}(a)} !} \quad p_{0}^{n_{0}} \prod_{1 \leq a \leq K: \boldsymbol{\psi}(a) \geq 1} p_{a}^{n_{\boldsymbol{\psi}(a)}}
$$

which, since the product over $a$ in the denominator of the multinomial term is equal to $\prod_{j=1}^{J}\left(n_{j} !\right)^{r_{j}}$, which is a constant, is proportional to (as a function of $\psi$ and $\theta$ )

$$
\frac{1}{n_{0} !} \quad p_{0}^{n_{0}} \prod_{1 \leq a \leq K: \boldsymbol{\psi}(a) \geq 1} p_{a}^{n_{\boldsymbol{\psi}(a)}} .
$$

The observed data likelihood is the sum over all mappings $\psi$ allowed by the constraints $\mathbf{C 1}$ and $\mathbf{C 2}$ of the full data likelihood. Note that $n_{0}$ occurs in the multinomial factor in the full data likelihood as well as as a power of $p_{0}$, and that $n_{0}$ depends on $\boldsymbol{\psi}$.

B.4. The moves. We will define a random walk on the set of all mappings $\boldsymbol{\psi}$ allowed by the constraints $\mathbf{C} 1$ and $\mathbf{C 2}$. It will be a Markov process with the set of mappings $\boldsymbol{\psi}$ as the (huge) state-space; the graph of possible transitions between states will however be sparse. Inspection of the likelihood (11) suggests two kinds of moves: $(i)$ An exchange move: exchanging the values of $\boldsymbol{\psi}(a)$ and $\boldsymbol{\psi}(b)$ for a chosen pair of different non-blob population species $a$ and $b$ such that $\boldsymbol{\psi}(a) \neq 0, \boldsymbol{\psi}(b) \neq 0$, and $\boldsymbol{\psi}(a) \neq \boldsymbol{\psi}(b)$, and (ii) A blob move: increasing or decreasing $n_{0}$ by one by choosing an $a$ such that $\boldsymbol{\psi}(a)=0$ or $\boldsymbol{\psi}(a)=1$ and exchanging the value 0 of $\boldsymbol{\psi}(a)$ for 1 or vice-versa.

Notice that these moves are not always possible. 
Lemma 1. (i): If $J \geq 3$ an exchange move is always possible. (ii): If $S>0$, where $S=r_{1}$ is the number of singletons, and $K>N$, where $N=\sum_{j=2}^{J} r_{j}$ is the number of non-singletons, a blob move is always possible.

Proof. (i): If we cannot find distinct $a, b$ with $\boldsymbol{\psi}(a) \neq 0, \boldsymbol{\psi}(b) \neq 0$, and $\boldsymbol{\psi}(a) \neq \boldsymbol{\psi}(b)$, an exchange move is impossible. However, as long as $J \geq 3$ there are at least two non-blob species observed a different number of times, and an exchange move is always possible.

(ii): It is always possible either to increase or to decrease $n_{0}$ but it is not always possible to do both, since there is a minimum value, which can only be increased, and a maximum value, which can only be decreased (unless the minimum and maximum possible values of $n_{0}$ coincide).

The maximum possible value of $n_{0}$, the number of times a blob species is observed, is the number of singletons $S$ in the sample, and it is feasible to let every singleton correspond to a blob species.

To determine the minimal value, define $L=N+S$, the length of the observed partition of $T$. The number of population species $a$ associated by $\boldsymbol{\psi}$ with singletons, i.e. such that $\boldsymbol{\psi}(a)=1$, cannot exceed the total number of singletons $S$ but it also cannot exceed $K-N$. It can equal the minimum of these two numbers. Thus the lower bound on $n_{0}$ is given by the requirement $S-n_{0} \leq \min (S, K-N)$, which is equivalent to $-n_{0} \leq \min (0, K-L)$, which is equivalent to $n_{0} \geq \max (0, L-K)$.

In summary, $\max (0, L-K) \leq n_{0} \leq S$ and therefore as long as $\max (0, L-$ $K)<S$ or equivalently $S>0$ and $L-K<S$, thus $K>N$, a blob move is always possible.

An exchange move defined by choice of a pair $(a, b)$ is its own reverse; and a blob move defined by choice of a single $a$ is its own reverse too. Moreover the number of candidate pairs $(a, b)$ for an exchange move is the same before and after the move. The number of candidates $a$ for a blob move is also the same before and after the move, except perhaps when $n_{0}$ is minimal or maximal. We shall further investigate these extreme cases later.

B.4.1. Exchange moves. For an exchange move we pick uniformly at random distinct $a$ and $b$ such that $\boldsymbol{\psi}(a) \neq 1, \boldsymbol{\psi}(b) \neq 1, \boldsymbol{\psi}(a) \neq \boldsymbol{\psi}(b)$. The Metropolis factor follows from the formula (11) for the full data likelihood. The move would convert the factor $p_{a}^{n_{\psi(a)}} p_{b}^{n_{\psi(b)}}$ into $p_{a}^{n_{\psi(b)}} p_{b}^{n_{\psi(a)}}$. The loga- 
rithm of the ratio of the full data likelihood "after" to "before" equals

$$
\begin{gathered}
\left(n_{\boldsymbol{\psi}(a)} \log p_{b}+n_{\boldsymbol{\psi}(b)} \log p_{a}\right)-\left(n_{\boldsymbol{\psi}(a)} \log p_{a}+n_{\boldsymbol{\psi}(b)} \log p_{b}\right) \\
=\left(n_{\boldsymbol{\psi}(a)}-n_{\boldsymbol{\psi}(b)}\right)\left(\log p_{b}-\log p_{a}\right) .
\end{gathered}
$$

Thus we draw $Z$ from the standard exponential distribution and accept the move if and only if, $\operatorname{since} \exp (-Z)$ is Unif $[0,1]$-distributed,

$$
-Z \leq\left(n_{\boldsymbol{\psi}(a)}-n_{\boldsymbol{\psi}(b)}\right)\left(\log p_{b}-\log p_{a}\right) .
$$

If the right hand side of (12) is positive, its exponent is larger than 1 , and the move is accepted. If the right hand side of (12) is negative, its exponent lies between 0 and 1 , and hence the move is accepted with probability equal to this exponent.

B.4.2. Blob moves. In order to describe a blob move we separate between the three cases where (i) $n_{0}$ is equal to its minimal value, $\max (0, L-K)$, or (ii) maximal value, $S$, or (iii) is somewhere in between.

If $n_{0}=\max (0, L-K)$, we pick a population species uniformly at random from the set $\{a: \psi(a)=1\}$. If $n_{0}=S$, we pick a population species uniformly at random from the set $\{a: \psi(a)=0\}$. When neither extreme case holds, we pick a sample species uniformly at random from the set $A(\boldsymbol{\psi})=$ $\{a: \boldsymbol{\psi}(a)=0$ or $\boldsymbol{\psi}(a)=1\}$.

However when $n_{0}=S$, there actually are no $a$ with $\psi(a)=1$, so the rule prohibiting us to pick one of such $a$ in this case is superfluous. Similarly, if $n_{0}=L-K \geq 0$ then there are no $a$ with $\boldsymbol{\psi}(a)=0$, and again the prohibition on picking such $a$ in this case is superfluous. Thus the rule for picking $a$ is simpler than first appeared: We always pick a population species uniformly at random from the set $A(\boldsymbol{\psi})$. The number of species in $A(\boldsymbol{\psi})$ is $K-N$, except when $L<K$ and $n_{0}=0$, in which case $A(\boldsymbol{\psi})=\{a: \boldsymbol{\psi}(a)=1\}$ and then the number of species is $S<K-N$.

After the random choice of a species $a$ from $A(\psi)$, the proposed move is to exchange the value of $\boldsymbol{\psi}(a)$ from 0 to 1 or vice-versa. In the first case $n_{0}$ is decreased by one, a factor $p_{a}^{1}$ gets added to the product of probabilities in (11), and the logarithm of the Metropolis contribution to the acceptance criterion is $\log p_{a}-\log p_{0}+\log n_{0}$. In the second case $n_{0}$ is increased by one, a factor $p_{a}^{1}$ gets deleted, and the logarithm of the Metropolis contribution to the acceptance criterion is $\log p_{0}-\log p_{a}-\log \left(n_{0}+1\right)$.

Recall that the Hastings factor in the Metropolis-Hastings algorithm is the ratio of the probabilities of the reverse move to the forward move. We have seen that, with one exception, the number of choices for $a$ is equal, 
both before and after the move, to $K-N$, so in general there is no Hastings contribution. The exceptional case is when $L<K$, and $n_{0}=0$ and is about to be increased by 1 (because we picked $a$ with $\psi(a)=1$ ), or $n_{0}=1$ and is about to be decreased by 1 (because we picked $a$ with $\psi(a)=0$ ). In these two cases the number of choices for the forward move and the reverse move are $S$ and $K-N>S$, and vice versa.

This means that if for a blob move we have picked $a$ with $\psi(a)=1$, the move (put $\boldsymbol{\psi}(a)=0$ ) is accepted if and only if

$$
-Z \leq \log p_{0}-\log p_{a}-\log \left(n_{0}+1\right) .
$$

(where $Z$ is a standard exponential random variable), except when $L<K$ and $n_{0}=0$, when the acceptance criterium is

$$
-Z \leq \log p_{0}-\log p_{a}-\log (K-N)+\log S
$$

If on the other hand we have picked $a$ with $\boldsymbol{\psi}(a)=0$, the move (put $\psi(a)=1)$ is accepted if and only if

$$
-Z \leq \log p_{a}-\log p_{0}+\log n_{0} .
$$

except when $L<K$ and $n_{0}=1$, when the acceptance criterium is

$$
-Z \leq \log p_{a}-\log p_{0}+\log (K-N)-\log S .
$$

B.5. The SA-EM. We next describe the "statistical part" of the SAEM algorithm. We use it to make a so called "stochastic approximation" of the conditional expectation of an underlying full data sufficient statistic given the actually observed data at the current parameter estimates, in the E step, and then to re-estimate the parameters by maximum likelihood using the current approximation of the full data sufficient statistic, in the M step.

A suitable choice for the sufficient statistic is the vector $\mathbf{g}$ of relative frequencies $g_{a}, 0 \leq a \leq K$, of the underlying population species in our sample of size $T$. Given the vector $\mathbf{f}$ of observed sample species distinct relative frequencies $f_{j}=n_{j} / T, 1 \leq j \leq J$, and given a realisation of the "missing" map $\boldsymbol{\psi}$, the underlying population relative frequencies are uniquely determined, for $1 \leq a \leq K$,

$$
\begin{aligned}
& g_{a}=f_{j}, \quad \text { if } \boldsymbol{\psi}(a)=j \geq 1 \\
& g_{a}=0, \quad \text { if } \boldsymbol{\psi}(a)=0 \\
& g_{0}=\frac{n_{0}}{T}
\end{aligned}
$$


At any point in the iterations we have a running estimate, denoted by $\boldsymbol{\mu}=\left(\mu_{a}\right)_{0 \leq a \leq K}$, of the conditional expectation of the vector $\mathbf{g}$ given the observed data $(\mathbf{n}, \mathbf{r})$. The expectation is taken under the current estimate of the vector of probabilities $\boldsymbol{\theta}=\left(p_{a}\right)_{0 \leq a \leq K}$. We generate a new realisation of $\mathbf{g}$ as just defined, thought to be a realisation from the distribution of $\mathbf{g}$ given $(\mathbf{n}, \mathbf{r})$ again under the current estimate of the parameter $\boldsymbol{\theta}$.

The stochastic approximation update is to replace the current estimate $\mathbf{m}$ of the conditional expectation of $\mathbf{g}$ given $(\mathbf{n}, \mathbf{r})$ under $\boldsymbol{\theta}$ by a weighted average of its current value and the current realisation $\mathrm{g}$ drawn from the distribution of $\mathbf{g}$ given $(\mathbf{n}, \mathbf{r})$ under $\boldsymbol{\theta}$ : replace $\boldsymbol{\mu}$ by $(1-\gamma) \boldsymbol{\mu}+\gamma \mathbf{g}$ where the weight $\gamma$ will be a function of the iteration number, which we denote by $k$.

These weights should satisfy $\sum_{k} \gamma_{k}=\infty, \sum_{k} \gamma_{k}^{2}<\infty$, cf. [1]. Many authors propose to take $\gamma_{k}=1 / k$ but we found that $\gamma_{k}=1 / k^{2 / 3}$ worked better. For small test problems, we found that an initial value of $k=k_{0}=$ 1000 gave good results in conjunction with $\gamma_{k}=1 / k^{2 / 3}$.

In the E-step we take the current value of the vector $\boldsymbol{\mu}=\left(\mu_{a}\right)_{0 \leq a \leq K}$ and maximise the $\log$ likelihood $\sum_{0 \leq a \leq K} \mu_{a} \log p_{a}$ subject to the constraints $p_{1} \geq p_{2} \geq \ldots p_{K} \geq 0, p_{0} \geq 0, \sum_{0 \leq a \leq K} p_{a}=1$. This is equivalent to taking $p_{1} \geq \cdots \geq p_{K}$ as a (version) of the isotonic (decreasing) regression of the vector $\left(\mu_{a}: 1 \leq a \leq K\right)$ which can be found using a modification of the wellknown pool adjacent violators algorithm, supplemented with the assignment $p_{0}=\mu_{0}$, see [3] and the comments in the discussion.

Apart from the initialisation of $k$, also a realization of the mapping $\boldsymbol{\psi}$, an value of $\boldsymbol{\theta}$, and a value of $\boldsymbol{\mu}$ need to be initialized. Since at convergence of the algorithm, $\boldsymbol{\mu}$ and $\boldsymbol{\theta}$ will be equal to one another, it also makes sense to initialise them equal to one another. A neutral initial guess for $\theta$ would be a defective uniform probability distribution on $\{1, \ldots, K\}$ supplemented with a not too small positive mass $p_{0}$ for the blob.

We initialise $\boldsymbol{\psi}$, thought of as a vector, by assigning its first $r_{J}$ components all with the value $J$, the next $r_{J}-1$ with the value $J-1$, and so on, until we get to the $r_{2}$ components assigned with the value 2 ; all remaining components are assigned the value 0 . Thus, under $\boldsymbol{\psi}$, a more frequently observed sample species has a larger population probability than a less frequently observed sample species, and all singletons are actually blob species.

\section{APPENDIX C: AN ALGORITHM FOR ESTIMATING A DECREASING MULTINOMIAL PROBABILITY WITH LOWER BOUND}

In this appendix we present an algorithm for bounded isotonic regression and prove its convergence. 
C.1. The algorithm and its convergence. Assume we have observations $\left(x_{1}, \ldots, x_{n}\right)$ of a multinomial random variable $\operatorname{Multi}(n, p)$ where $n=\sum_{i=1}^{k} x_{i}$ with $p=\left(p_{1}, \ldots, p_{k}\right)$ a vector a numbers $p_{i} \in[0,1]$ such that $\sum_{i=1}^{k} p_{i}=1$ and $p_{1} \geq \ldots \geq p_{k}$. Assume that the vector $p$ is unknown and assume also that for a given constant $0<c<1 / k$ we know that $p_{k} \geq c$. The goal is then to estimate $p$ under the assumption that

$$
A^{(c)}(p)=\left\{p_{1} \geq \ldots \geq p_{k} \geq c\right\}
$$

holds. Note that the restrictions (17) can be written as

$$
A^{(c)}(p)=\cup_{j=1}^{k} A_{j}^{(c)}(p)
$$

with

$$
\begin{aligned}
& A_{j}^{(c)}(p)=\left\{p_{1} \geq \ldots \geq p_{j}\right\} \cap\left\{p_{j+1}=\ldots=p_{k}\right\} \cap\left\{p_{j} \geq c\right\} \\
& A_{j}^{(c)}(p) \subset A_{j+1}^{(c)}(p), \text { for all } p, j \text { and } c .
\end{aligned}
$$

Let $\mathcal{F}_{c}=\left\{q \in[0,1]^{k}: \sum_{i=1}^{k} q_{i}=1, q_{1} \geq \ldots \geq q_{k} \geq c\right\}$. Define the likelihood and $\log$ likelihood as

$$
\begin{aligned}
L(p) & =\frac{n !}{x_{1} ! \cdots x_{k} !} p_{1}^{x_{1}} \cdots p_{k}^{x_{k}} \\
\log L(p) & \sim \sum_{i=1}^{n} x_{i} \log \left(p_{i}\right)=: l(p)
\end{aligned}
$$

and define the order restricted mle $\hat{p}^{(c)}$ with lower bound at $c$ as (where appropriate we suppress the explicit dependence of the estimate on $c$ in the notation, and thus write $\hat{p}=\hat{p}^{(c)}$ )

$$
\hat{p}^{(c)}=\left(\hat{p}_{1}, \ldots, \hat{p}_{k}\right)=\operatorname{argmax}_{q \in \mathcal{F}_{c}} \sum_{i=1}^{k} x_{i} \log q_{i}=\operatorname{argmax}_{q \in \mathcal{F}_{c}} l(q) .
$$

The linear restriction $\sum_{i=1}^{k} q_{i}=1$ can be taken care of by introducing a Lagrange multiplier, so that the optimization is equivalent to maximization of

$$
\tilde{l}(q, \lambda)=\sum_{i=1}^{n} x_{i} \log \left(q_{i}\right)-\lambda\left(\sum_{i=1}^{k} q_{i}-1\right)
$$

with respect to $q$, over the set $\mathcal{F}_{c}=\left\{q \in[0,1]^{k}: q \in A^{(c)}(q)\right\}$. 
Note that with $\mathcal{F}_{c, j}=\left\{q \in[0,1]^{k}: q \in A_{j}^{(c)}(q)\right\}$, by (17), (18), (19) we obtain

$$
\begin{aligned}
\mathcal{F}_{c} & =\cup_{j=1}^{k} \mathcal{F}_{c, j}, \\
\mathcal{F}_{c, j} & \subset \mathcal{F}_{c, j+1}, \text { for all } c, j .
\end{aligned}
$$

This shows that (since $\mathcal{F}_{c, k}=\mathcal{F}_{c}$, this is only the definition of the MLE again)

$$
\hat{p}^{c}=\operatorname{argmax}_{q \in \mathcal{F}_{c, k}, \lambda} \tilde{l}(q, \lambda) .
$$

We will maximize $\tilde{l}$ over $\mathcal{F}_{c}$, by going through the sets $\mathcal{F}_{c, k}, \mathcal{F}_{c, k-1} \ldots$ Note that the function $l$ is strictly concave, since it is a composition of the strictly concave function $\log$ and a linear function, and the set $\mathcal{F}_{c}$ over which one wants to optimize $l$ is a closed convex set, since $\mathcal{F}_{c}$ is an intersection of closed convex cones and a hyperplane. This implies that there is a global solution $\hat{p}^{c}$, that it is unique and that $\hat{p}^{c}$ lies in $\mathcal{F}_{c}$.

We present an iterative algorithm for obtaining the solution; this goes via a sequence $\hat{p}^{(1)}, \hat{p}^{(2)}, \ldots, \hat{p}^{(j)}$, with $j \in\{1, \ldots, k\}$ a random index, and such that $\hat{p}^{(j)}=\hat{p}^{c}$ so that the algorithm converges, in a finite number of steps.

\section{Algorithm 1. (Bounded isotonic regression)}

1. Let

$$
\hat{p}^{(1)}=\operatorname{argmax}_{q \in \mathcal{F}_{0, k}, \lambda} \tilde{l}(q, \lambda),
$$

be the unrestricted estimator. Then if $\hat{p}_{k}^{(1)} \geq c$, we are done, and the algorithm has converged with $j=1$.

2. If not, so if $\hat{p}_{k}^{(1)}<c$, we define the next element $\hat{p}^{(2)}$ in the sequence as follows: Let $\hat{p}_{k}^{(2)}=c$. Define

$$
\left(\hat{p}_{1}^{(2)}, \ldots, \hat{p}_{k-1}^{(2)}\right)=\operatorname{argmax}_{q_{1} \geq \ldots \geq q_{k-1} \geq 0, \lambda} \tilde{l}\left(q_{1}, \ldots, q_{k-1}, c, \lambda\right) .
$$

If $\hat{p}_{k-1}^{(2)} \geq c$ we are done, and the algorithm has converged with $j=2$.

3. If not, so if $\hat{p}_{k-1}^{(2)}<c$, we define the next element $\hat{p}^{(3)}$ in the sequence by: Let $\hat{p}_{k-1}^{(3)}=\hat{p}_{k}^{(3)}=c$. Define

$$
\left(\hat{p}_{1}^{(3)}, \ldots, \hat{p}_{k-2}^{(3)}\right)=\operatorname{argmax}_{q_{1} \geq \ldots \geq q_{k-2} \geq 0, \lambda} \tilde{l}\left(q_{1}, \ldots, q_{k-2}, c, c, \lambda\right) .
$$

4. Continued in the obvious way, until convergence. 
To prove that the algorithm converges we need the following simple result that has independent interest and is stated for completeness.

LEMMA 2. Assume that $h$ is a function, concave over a convex set $I \subset$ $\mathbb{R}^{k}$, with the set $I$ defined by inequalities and linear restrictions. Then, for any integer $0<b<k$, the function

$$
g\left(x_{b+1}, \ldots, x_{k}\right)=\sup _{\left(x_{1}, \ldots, x_{b}\right):\left(x_{1}, \ldots, x_{b}, x_{b+1}, \ldots, x_{k}\right) \in I} h\left(x_{1}, \ldots, x_{b}, x_{b+1}, \ldots, x_{k}\right)
$$

is concave over the convex set

$$
I_{\bar{b}}=\left\{\left(x_{b+1}, \ldots, x_{k}\right) \in \mathbb{R}^{b}:\left(x_{1}, \ldots, x_{b}, x_{b+1}, \ldots, x_{k}\right) \in I\right\} .
$$

Proof. By assumption $I$ can be written as an intersection of sets of the form $\left\{x=\left(x_{1}, \ldots, x_{k}\right): x_{j} \leq x_{l}\right\}$ (closed convex cones) and $\{x=$ $\left.\left(x_{1}, \ldots, x_{k}\right): \sum_{i=1}^{k} a_{i} x_{i}=c\right\}$ (a hyperplane) for some real numbers $a_{i}, i=$ $1, \ldots, k$ and $c$. Recall that by definition $h$ is concave over $I$ if it's epigraph is a convex set. We use this repeatedly:

The individual maximization $h$ as above means projecting the epigraph of $h$ on the faces $\left\{x=\left(x_{1}, \ldots, x_{k}\right): x_{j} \leq x_{l}\right\}$. Each such projection (i.e. maximization) gives a convex set (in $\mathbb{R}^{k}$ ) and the intersection of them is convex, and thus the epigraph of a concave function over $I_{\bar{b}}$, which shows that $g$ is concave.

THEOREM 1. If $0<c<1 / k$, the algorithm above converges to the global maximum $\hat{p}^{(c)}$.

Proof. We want to maximize $\tilde{l}$ over $\mathcal{F}_{c}$, which we do by stepwise going through the sets $\mathcal{F}_{c, 1}, \mathcal{F}_{c, 2}, \ldots, \mathcal{F}_{c, k}$ starting with $\mathcal{F}_{c, k}$.

If after step $(i), \hat{p}_{k}^{(1)} \geq c$, then since $\mathcal{F}_{c} \subset \mathcal{F}_{0}$ the maximum over $\mathcal{F}_{0}$ (i.e. $\hat{p}_{k}^{(1)}$ ) is equal to the maximum over $\mathcal{F}_{c}$ and we are done.

Assume that we are not done and instead that $\hat{p}_{k}^{(1)}<c$. Define the function

$$
g_{1}(u)=\max _{q_{1} \geq \ldots \geq q_{k-1} \geq u, \lambda} \tilde{l}\left(q_{1}, \ldots, q_{k-1}, u, \lambda\right) .
$$

By the above lemma $g_{1}$ is a concave function. Thus it attains it's maximum at some point, which is $\hat{p}_{k}^{(1)}$. From the assumption $c>\hat{p}_{k}^{(1)}$ and by the concavity of $g_{1}$ we have that $g_{1}(u)<g_{1}(c)$ for any $u>c>\hat{p}_{k}^{(1)}$, i.e. that for

$$
\max _{q_{1} \geq \ldots \geq q_{k-1} \geq u, \lambda} \tilde{l}\left(q_{1}, \ldots, q_{k-1}, u, \lambda\right)<\max _{q_{1} \geq \ldots \geq q_{k-1} \geq c, \lambda} \tilde{l}\left(q_{1}, \ldots, q_{k-1}, c, \lambda\right) .
$$


This implies that, when maximizing under the restriction $\mathcal{F}_{c, k}$, we can actually fix $u=c$ and look for

$$
\operatorname{argmax}_{q_{1} \geq \ldots \geq q_{k-1} \geq c, \lambda} \tilde{l}\left(q_{1}, \ldots, q_{k-1}, c, \lambda\right) .
$$

We do this as follows: In step $(i i)$ we replace the lower bound $q_{k-1} \geq c$ by $q_{k-1} \geq 0$. Thus we are looking over a larger set. If $\hat{q}_{k-1}^{(2)} \geq c$ we are done. If not, so if $c>\hat{q}_{k-1}^{(2)}$, we study the function

$$
g_{2}(u)=\max _{q_{1} \geq \ldots \geq q_{k-1} \geq 0, \lambda} \tilde{l}\left(q_{1}, \ldots, q_{k-1}, c, \lambda\right) .
$$

An application of Lemma 2 shows that $g_{2}$ is a concave function, it's maximum is attained at $\hat{q}_{k-1}^{(2)}$, and by concavity $g_{2}(u)<g_{2}(c)$ for any $u<c<$ $\hat{q}_{k-1}^{(2)}$. Thus when maximizing under the restriction $\mathcal{F}_{c, k-1}$, we may fix $u=c$ and look for

$$
\operatorname{argmax}_{q_{1} \geq \ldots \geq q_{k-2} \geq c, \lambda} \tilde{l}\left(q_{1}, \ldots, q_{k-2}, c, c, \lambda\right) .
$$

We do this as follows: In step (iii), replace the lower bound $q_{k-2} \geq c$ by $q_{k-2} \geq 0$, and so on.

(iv) Continue until convergence.

This scheme is clearly finite, and will terminate with $\hat{q}_{k-j}^{(j+1)} \geq c$ for some $j<k$, since $c<1 / k, p_{1} \geq \ldots \geq p_{k}$ and $\sum_{i=1}^{k} p_{k}=1$.

Furthermore, the scheme searches for maxima, over the sets $\mathcal{F}_{c, k} \backslash \mathcal{F}_{c, k-1}$, $\mathcal{F}_{c, k-1} \backslash \mathcal{F}_{c, k-2}, \ldots$. If it stops at an index $j$, that means that $\hat{p}_{k-j}^{(j+1)} \geq c$ and that $\hat{p}_{k-j}^{(j+1)}$ is obtained as a maximum over the set $\mathcal{F}_{0, k-j}$, which is also a maximum over the set $\mathcal{F}_{c, k-j}$, and we will have searched over the set

$$
\left(\cup_{i=1}^{j} \mathcal{F}_{c, k-i+1} \backslash \mathcal{F}_{c, k-i}\right) \cup \mathcal{F}_{c, k-j}=\mathcal{F}_{c}
$$

i.e. the solution is a global solution. This ends the proof of convergence.

C.2. Implementation of the algorithm. We now present a numerical implementation of Algorithm 1 in $\mathrm{C} / \mathrm{C}++$. Recall that in the bounded isotonic regression $p_{1} \geq \ldots \geq p_{k} \geq c$. The algorithm uses the standard isotonic regression with $p_{1} \geq \ldots \geq p_{k} \geq 0$, whose implementation is called isoreg ( ) and can be found in the literature [3] (see also its implementation in $\mathrm{R})$. The implementation of Algorithm 1 thus reads: 


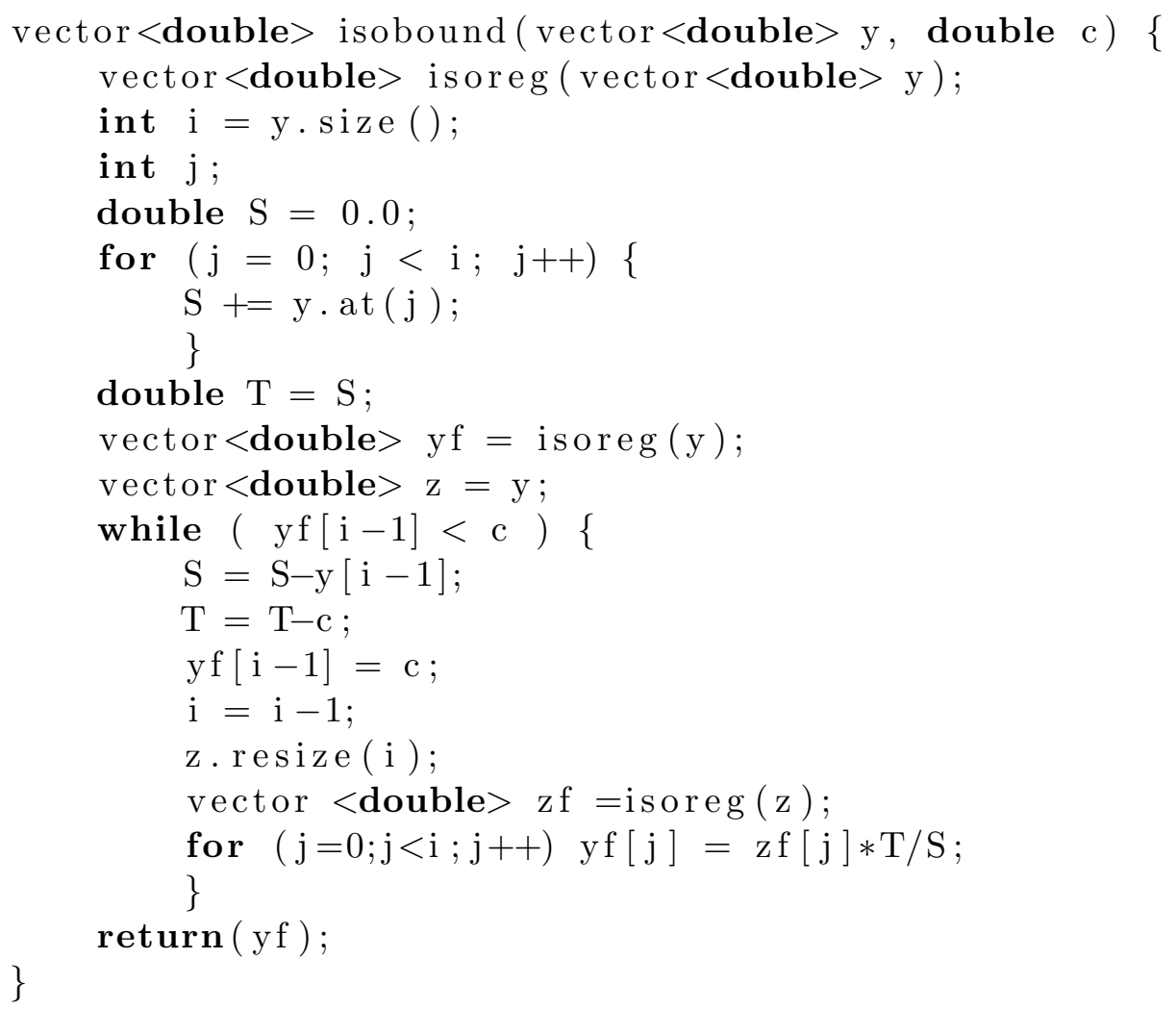

\section{REFERENCES}

[1] B. Delyon, M. Lavielle, and E. Moulines. Convergence of a stochastic approximation version of the EM algorithm. Ann. Statist., 27(1):94-128, 1999.

[2] A. Orlitsky and S. Pan. The maximum likelihood probability of skewed patterns. In IEEE International Symposium on Information Theory, 2009.

[3] T. Robertson, F.T. Wright, and R.L. Dykstra. Order Restricted Statistical Inference. John Wiley \& Sons Inc., New York, 1988.

Centre for Mathematical Sciences,

LUND UNIVERSITY,

Box 118, 22100 Lund,

SWEDEN

E-MAIL: dragi@maths.lth.se
Mathematical Institute, LEIDEN UNIVERSITY

Niels Bohrweg 1, 2333 CA Leiden, The Netherlands

E-MAIL: gill@math.leidenuniv.nl

Department of Materials,

PARKS ROAD, OX1 3PH, OxFord, UK

E-MAIL: stefan.zohren@materials.ox.ac.uk 